\title{
Review Article \\ Mesenchymal Stem Cells Improve Healing of Diabetic Foot Ulcer
}

\author{
Yue Cao, Xiaokun Gang, Chenglin Sun, and Guixia Wang \\ Department of Endocrinology and Metabolism, The First Hospital of Jilin University, Changchun 130021, China \\ Correspondence should be addressed to Guixia Wang; gwang168@jlu.edu.cn
}

Received 24 September 2016; Accepted 28 February 2017; Published 12 March 2017

Academic Editor: Ruozhi Zhao

Copyright (C) 2017 Yue Cao et al. This is an open access article distributed under the Creative Commons Attribution License, which permits unrestricted use, distribution, and reproduction in any medium, provided the original work is properly cited.

\begin{abstract}
Mesenchymal stem cells (MSCs), an ideal cell source for regenerative therapy with no ethical issues, play an important role in diabetic foot ulcer (DFU). Growing evidence has demonstrated that MSCs transplantation can accelerate wound closure, ameliorate clinical parameters, and avoid amputation. In this review, we clarify the mechanism of preclinical studies, as well as safety and efficacy of clinical trials in the treatment of DFU. Bone marrow-derived mesenchymal stem cells (BM-MSCs), compared with MSCs derived from other tissues, may be a suitable cell type that can provide easy, effective, and cost-efficient transplantation to treat DFU and protect patients from amputation.
\end{abstract}

\section{Introduction}

In recent years, with the rapid economic growth and the change of diet structure, the incidence of diabetes mellitus (DM) increased gradually $[1,2]$. According to epidemiological surveys, diabetes had spread to 422 million people worldwide by 2014 [3]. And the number of patients with DM may be more than 360 million in 2030 [4]. In addition, huge economic burden from treatment and care of DM is laid on the patients and society [5]. In the US, the cost on diagnosis of DM in 2012 was $\$ 245$ billion, with a $41 \%$ increase compared with the expenditure in 2007 [6].

There is an alarming increase in the macro- and microvascular complications secondary to DM, in which DFU is one of the most common complications. Statistical data has demonstrated that more than a quarter of patients suffered from DFU [7]. According to the International Working Group on Diabetic Foot, risk of DFU increases with increasing age, long history of DM, and high HbAlc [8]. DFU is a complex and severe clinical problem that can lead to subsequent limb amputation. The amputation rate of DM was $19.03 \%$ in China in 2015 [9]. At present, patients with DFU are still bearing a high risk of amputation and high costs of treatment and care [10]. In summary, DFU is one of the leading factors that threaten human health and aggravate economic burden [11].

Diverse sources and the potential of self-renewing and multidifferentiation are main characteristics of stem cells, which make stem cell therapy a new alternative to repair and regenerate tissues. Nowadays, a growing number of diseases can be improved via wide applications of stem cell transplantation, such as congenital cataract [12], diabetic retinopathy and keratopathy [13], myocardial infarction [14], ocular surface burns $[15,16]$, serious skin burns $[17,18]$, Parkinson's disease [19], Huntington's disease [20], and especially DFU [21]. Accumulating evidence has pointed out that mesenchymal stem cells (MSCs) may enhance wound healing [22-24] and be served as a cell source for many tissue engineering applications including bone regeneration [25], cartilage regeneration [26-28], myocardial regeneration [29], neurogenesis [30, 31], inflammatory bowel diseases [32], and DFU $[33,34]$. MSCs exist in many tissues, for example, bone marrow [35, 36], umbilical cord [37, 38], placenta [39, 40], adipose tissue [36, 41-43], gingiva [44, 45], oral mucosa [46], amniotic fluid [47], and brain [48]. However, the appropriate cell type and selection between autologous or allogeneic MSCs are yet to be discussed. Therefore, the present article reviews the roles of autologous or allogeneic MSCs derived from different tissues in wound healing of DFU.

\section{BM-MSCs and DFU}

2.1. Intrinsic Property. Bone marrow is one of the most common tissues from which MSCs can be acquired. BMMSCs have no immunologic restriction and do not stimulate 


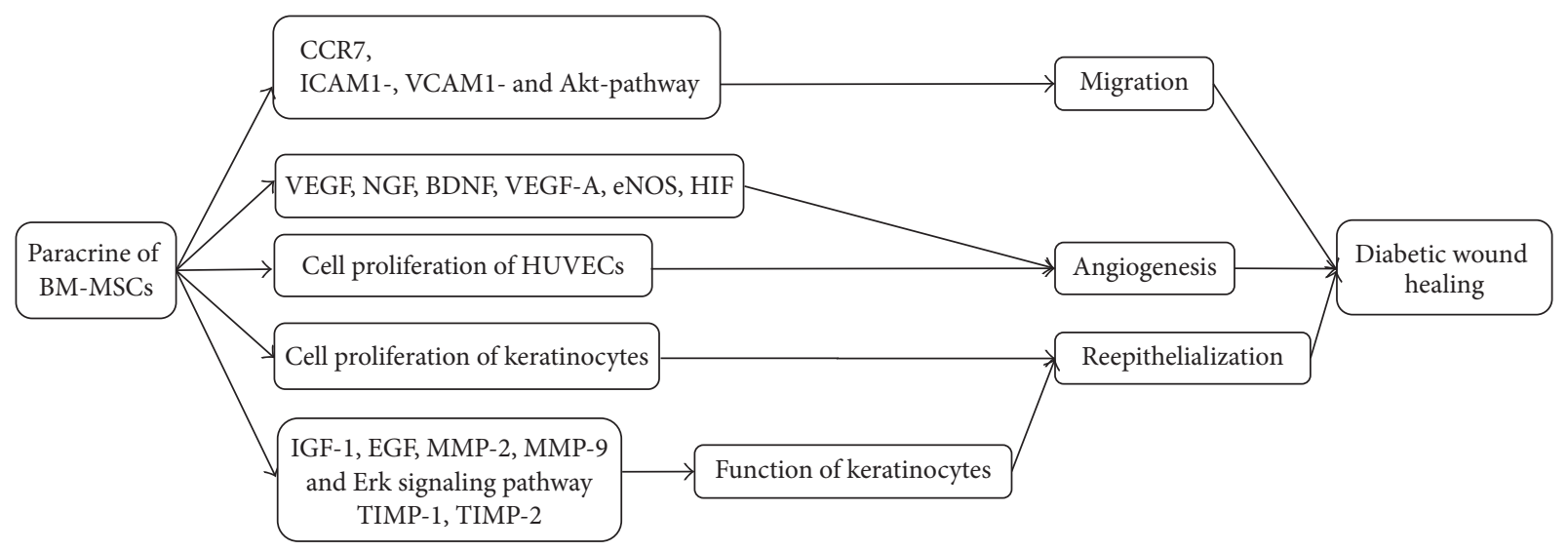

Figure 1: Mechanism of BM-MSCs for treatment of DFU. BM-MSCs can migrate and adhere via CCR7, ICAM1-, VCAM1-, and Aktdependent mechanism and enhance angiogenesis through increasing VEGF, NGF, BDNF, VEGF-A, eNOS, and HIF. Cell proliferation of HUVECs and keratinocytes plays significant role in angiogenesis and reepithelialization, respectively. Keratinocyte function is improved by regulating IGF-1, EGF, MMP-2, MMP-9, TIMP-1, TIMP-2, and Erk signaling pathway. CCR7: C-C chemokine receptor type 7, ICAM1: intercellular adhesion molecule 1, VCAM1: vascular adhesion molecule 1, VEGF: vascular endothelial growth factor, NGF: nerve growth factor, BDNF: brain-derived neurotrophic factor, VEGF-A: vascular endothelial growth factor A, eNOS: endothelial nitric oxide synthase, HIF: hypoxia inducible factor, IGF-1: insulin-like growth factor 1, EGF: epidermal growth factor, MMP-2: matrix metalloproteinase-2, MMP9: matrix metalloproteinase-9, TIMP-1: tissue inhibitor of metalloproteinase-1, and TIMP-2: tissue inhibitor of metalloproteinase-2.

alloreactivity because they have capability of escaping lysis by cytotoxic T-cell and natural killer (NK) cells, reducing the formation of cytotoxic lymphocytes [49], and suppressing Tcell-derived interferon-gamma (IFN- $\gamma$ ), as well as proliferation of T-cell and NK cells induced by cellular or humoral stimuli in vitro [50, 51]. Thus, BM-MSCs transplantation is a safe way for DFU, and intramuscular transplantation has been proved to have the best efficacy [22]. However, the number and differentiated potential of BM-MSCs decline with aging [52].

\subsection{Mechanism}

2.2.1. Paracrine. BM-MSCs can enhance the migration, angiogenesis, and reepithelialization via paracrine to accelerate wound repair.

Allogeneic BM-MSCs can migrate and home to the wound area [22] through expressing $\mathrm{C}-\mathrm{C}$ chemokine receptor type 7 (CCR7) [53] and adhere to endothelial cells (ECs) via intercellular adhesion molecule 1- (ICAM1-), vascular adhesion molecule 1- (VCAM1-), and Akt-dependent mechanism [54].

Wan and colleagues found that allogeneic BM-MSCs could promote angiogenesis and thicken granulation tissue by increasing the expression of vascular endothelial growth factor (VEGF) in diabetic rats [22]. O'Loughlin and colleagues indicated that allogeneic BM-MSCs seeded in a collagen scaffold could improve wound healing by augmenting angiogenesis in diabetic rabbit ear ulcer model [55]. In the study of diabetic mice, neurotrophin-3- (NT-3-) stimulated human BM-MSCs in the biological tissue material expressed VEGF, nerve growth factor (NGF), brain-derived neurotrophic factor (BDNF), and other vascular endothelial factors to upregulate angiogenesis and thicken granulation tissue [56]. Coincidentally, the conditioned medium of heme oxygenase-1- (HO-1-) overexpressing human BM-MSCs promoted the proliferation and migration of human umbilical vein endothelial cells (HUVECs) in vitro. Therefore, the complex of HO-1 -overexpressing human BM-MSCs and collagen biomaterials also could promote angiogenesis and stimulate wound cicatrization in the mice of diabetic ischemic ulcer [57].

In addition, allogeneic BM-MSCs prestimulated with EGF stimulate the neovascularization through the modulation of vascular endothelial growth factor A (VEGF-A), endothelial nitric oxide synthase (eNOS), hypoxia inducible factor (HIF), and VEGF/VEGF receptor pathways in diabetic mice, thereby enhancing the recovery of blood flow [54].

Reepithelialization in the wound is a consequence of cell proliferation and modification of keratinocyte functions. Allogeneic BM-MSCs shortened the duration of wound healing in diabetic foot ulcerations on the plantar skin of rats via the improvement of keratinocytes which had been mentioned in vitro [33]. It has been found that BM-MSCs isolated from rats not only promote human keratinocytes (HKCs) to produce cytokine including matrix metalloproteinase-2 (MMP-2), epidermal growth factor (EGF), and insulin-like growth factor 1 (IGF-1) [33], but also enhance the migration and proliferation of rat keratinocytes by upregulating MMP2 and matrix metalloproteinase-9 (MMP-9) and downregulating tissue inhibitor of metalloproteinase-1 (TIMP-1) and tissue inhibitor of metalloproteinase-2 (TIMP-2), as well as triggering Erk signaling pathway [58] (Figure 1).

2.2.2. Mobilization of Autologous Stem Cells. Iwamoto and colleagues demonstrated that autologous stem cells mobilized from bone marrow by systemic injections of granulocyte colony-stimulating factor (GCSF) improved wound 
bed preparation and accelerated healing in mice [59]; albeit the presence of BM-MSCs in mobilized stem cells was not identified in this study, they were shown to be mobilized by GCSF in previous study [60]. In Tatsumi et al. study, GCSF might promote bone marrow-derived stem cells to mobilize and migrate to the wound site and improve granulation tissue to enhance epithelialization, rather than exerting a direct effect on epithelialization of wounds in both mice and human without diabetes. However, GCSF was unable to enhance epidermal migration from the wound margins in $\mathrm{db} / \mathrm{db}$ diabetic mice with tail wounds.

The reasons are likely that diabetic microenvironment including hyperglycemia and persistence of inflammation may have an influence on population and function of endogenous stem cell. Compared to wild-type mice, $\mathrm{db} / \mathrm{db}$ diabetic mice possessed fewer MSCs, of which viability, homing capacity, and therapeutic capacity were impaired [61]. Clinical trials showed that the number of MSCs was decreased and the phenotype of MSCs was altered in patients with diabetic foot syndrome [62]. Based on minimal criteria to define human MSCs [63], MSCs are defined as positive for CD105, CD73, and CD90 and negative for CD45, CD34, $\mathrm{CD} 14$ or CD11b, $\mathrm{CD} 79 \alpha$ or CD19, and HLA-DR surface markers. However, $\mathrm{CD} 45^{-}, \mathrm{CD} 29^{+}$, and $\mathrm{CD} 90^{+}$MSCs were increased in subjects with diabetic foot syndrome [62]. Moreover, migration process was compromised as a result of less expression of adhesion molecules, such as ICAM1 and VCAM1 [54].

2.3. The Safety and Efficacy of Clinical Trials. In clinical trials, autologous transplantation of BM-MSCs can significantly ameliorate clinical parameters including decrease in wound size and increase in pain-free walking distance and maintain normal liver and renal function following intervention [64]. Leg perfusion is also sufficiently improved to minimize major amputations $[65,66]$. It has been discovered that autologous biograft in combination with BM-MSCs decreases wound size and increases dermal vascularity and thickness in patients with DFU [67].

At 6 weeks after intramuscular injection of autologous BM-MSCs, the ulcer healing rate of T2DM patients with bilateral critical limb ischemia (CLI) and foot ulcer increased significantly. After 24 weeks of follow-up, painless walking time, limb perfusion, ankle-brachial index (ABI), transcutaneous oxygen pressure $\left(\mathrm{TcO}_{2}\right)$, and magnetic resonance angiography (MRA) analysis were also improved significantly [34] (Table 1).

\section{Umbilical Cord Blood-Derived Mesenchymal Stem Cells (UCB-MSCs) and DFU}

3.1. Intrinsic Property. UCB-MSCs have a similar morphology, cell surface antigens, and the potential of differentiation into BM-MSCs and umbilical cord-derived mesenchymal stem cells (UC-MSCs) [42, 52, 68]. Additionally, UCB-MSCs have several advantages, such as short doubling time [69], long viable time, and anti-inflammatory activity [42]. Thus,
UCB-MSCs are considered as convenience and abundance seed cells for regenerative medicine.

\subsection{Mechanism}

3.2.1. Paracrine. Animal studies have indicated the ability of human UCB-MSCs to prevent or cure DFU via angiogenesis and the expression of nerve growth factor (NGF) in femoral nerve innervated gastrocnemius of diabetic foot ulceration rats [70]. In vitro, human UCB-MSCs might have capacity for diabetic wound healing by producing VEGF and basic fibroblast growth factor (bFGF) [71].

3.3. The Safety and Efficacy of Clinical Trials. It has been reported that transplantation of allogeneic UCB-MSCs injected into the quadriceps thigh muscles of individuals with DFU improves clinical profiles. All patients following allogeneic UCB-MSCs transplantation have decreased blood glucose, insulin dosage, levels of C-reactive protein (CRP), and tumor necrosis factor $\alpha$ (TNF- $\alpha$ ), as well as increased VEGF and the ratios of $\mathrm{CD} 4^{+} \mathrm{CD} 25$ (hi) FoxP3 ${ }^{+}$Treg/Th17 and $\mathrm{CD}_{4}^{+} \mathrm{CD} 25$ (hi) FoxP3 ${ }^{+}$Treg/Th1 cells. Moreover, the ratio of Treg/Th17 also had a correlation with the levels of VEGF and interleukin-6 (IL-6) detected in the plasma of patients [72].

However, a phase I study on patients with CLI indicated that intramuscular injection of allogeneic UCB-MSCs improved symptoms or clinical parameters with some side effects. Adverse events including whole body urticaria, diarrhea, oral ulceration, and elevation of serum creatinine level were observed in three patients; however, all conditions were resolved in short order [73].

Up to now, the application of UCB-MSCs for DFU is little. We consider that the extraction of UCB-MSCs involved in privacy and ethic may be a concern; meanwhile the cost in preservation of umbilical cord blood is very high (Table 1).

\section{MSCs Derived from Other Tissues}

Up to date, preclinical studies on adipose-derived mesenchymal stem cells (AMSCs), umbilical cord-derived mesenchymal stem cells (UC-MSCs), placenta-derived mesenchymal stem cells (PMSCs), and human amniotic fluid-derived stem cells (AF-MSCs) for diabetic wound healing have been reported, but no clinical trials have been reported. However, human gingiva-derived mesenchymal stem cells (GMSCs) are only investigated in excisional wound model, and the data are quite limited.

\subsection{AMSCs and Diabetic Wound Healing}

4.1.1. Intrinsic Property. Adipose tissue derived from the mesenchyme is widely distributed and easily isolated. AMSCs have high colony frequency and represent an attractive alternative source of pluripotent cells, whose characteristics are similar to BM-MSCs $[42,74]$.

4.1.2. Mechanism. In diabetic rats with dorsal full-thickness skin wound, allogeneic AMSCs injected subcutaneously in 


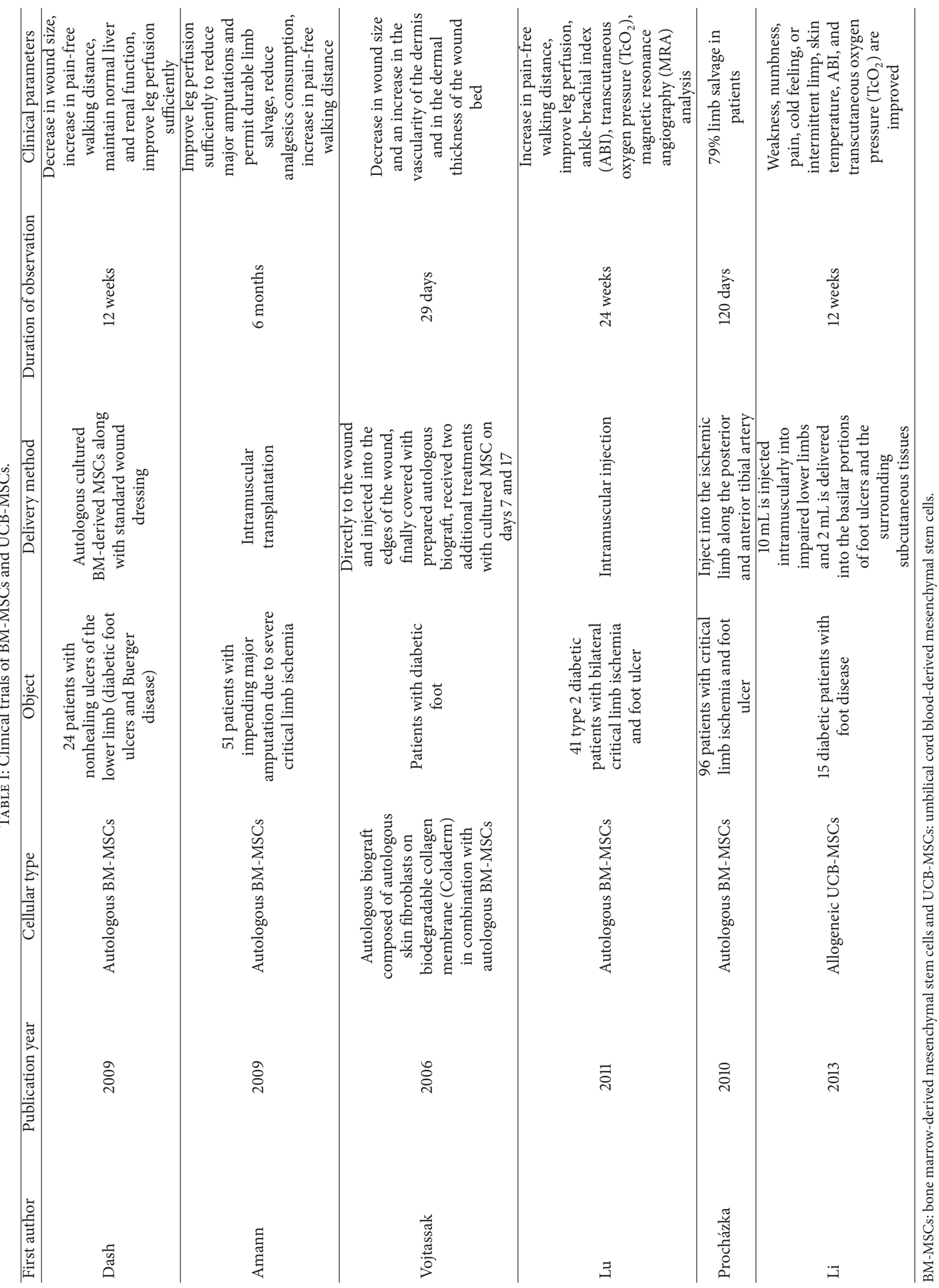


the wound margin stimulated neoangiogenesis and increased tissue regeneration through paracrine and autocrine mechanisms [75]. The data showed that allogeneic AMSCs migrated to the wound margin and increased angiogenesis via the activation of endothelial activity and neoangiogenic capacities by increasing VEGF and von Willebrand factor (vWF). Simultaneously, as a proliferating cell nuclear antigen, Ki-67, was up-regulated to promote cellular proliferation. The proinflammatory reaction was reduced through the expression of EGF, VEGF, and prolyl 4-hydroxylase ( $\mathrm{rPH}$ ). Consistent with this notion, allogeneic AMSCs were harvested from the inguinal fat of normal rats secreted large amounts of several angiogenic growth factors including VEGF, hepatocyte growth factor (HGF), transforming growth factor beta 1 (TGF- $\beta 1$ ), IGF-1, EGF, and keratinocyte growth factor (KGF) in vitro. In vivo, the transplantation of AMSCs sheets was created using cell-sheet technology accelerated wound healing and vascularization in full-thickness skin defects in Zucker diabetic fatty rats [76].

Additionally, direct injection of ASCs obtained from nondiabetic patients into full-thickness wound of diabetic mice model significantly increased the rate of wound closure [77]. In another study on diabetic mice, the new findings that silk fibroin patches cellularized with human adipose-derived MSCs (Ad-MSCs-SF) and silk fibroin patches decellularized with human adipose-derived MSCs (D-Ad-MSCs-SF) patches improved tissue regeneration and reduced the wound area through releasing angiogenic factors and collagen deposition stimulating molecules [78]. A decrease in the risk of transferring genetically mutated cells and the possibility of stimulating the immune system were the advantage of DAd-MSCs-SF patches, and decellularized patches could be prepared and stored for an extended period.

\subsection{UC-MSCs and Diabetic Wound Healing}

4.2.1. Intrinsic Properties. UC-MSCs are generally considered to be rich, safe, of short doubling time, and easy to collect [52]. Compared to BM-MSCs, it has been well documented that UC-MSCs have similar characteristics involving fibroblastic morphology, typical immunophenotypic markers, and multiple differentiation potential to BM-MSCs [79-82]. In addition, the trait of UC-MSCs has lower immunogenicity $[83,84]$.

4.2.2. Mechanism. In the study on DFU rats with UC-MSCs delivered through the left femoral artery, researchers found that UC-MSCs could specifically localize to the targeted area by detecting the expression of human leukocyte antigen typeI (HLA-1), a marker to track UC-MSCs in vivo.

Besides, UC-MSCs significantly reduced the size of foot ulcers and promoted epithelialization of ulcerated tissue via release of cytokeratin 19 from keratinocytes and formation of extracellular matrix [21]. In other studies of DFU rats, the data showed that administration of UC-MSCs contributed to improvement of vascular density $[85,86]$ and repair of wound and sensory functions [87] by the expression of VEGF, keratinocyte growth factor (KGF), platelet derived growth factor (PDGF), and brain-derived growth factor (BDGF).

\subsection{PMSCs and Diabetic Wound Healing}

4.3.1. Intrinsic Property. Placental tissue is readily available and can isolate a large number of MSCs for clinical application [88]. What is more, the morphology, size, surface phenotype, and immunosuppressive characteristics of PMSCs are similar to BM-MSCs, and the proliferation capability is better [39]. The best efficacy delivery is intraperitoneal injection [89].

4.3.2. Mechanism. In the research of diabetic Goto-Kakizaki (GK) rats, the experimental group showed that implanted PMSCs gathered to the wound tissue and differentiated into endothelial-like cells. Additionally, it has been found that PMSCs participate in angiogenesis in wound bed through secreting some proangiogenic molecules, such as VEGF, bFGF, and IGF-1, transforming growth factor- $\beta$ (TGF- $\beta$ ) and hepatocyte growth factor (HGF) [90].

4.4. AF-MSCs and Diabetic Wound Healing. Large numbers of human AF-MSCs can be easily harvested from as little as $2 \mathrm{~mL}$ of amniotic fluid [91]. Human AF-MSCs remain stable and show high proliferative capacity, multilineage differentiation potential, immunomodulatory activity, and lack of significant immunogenicity [92].

The transplantation of human AF-MSCs has been shown to accelerate wound healing by secreting factors [93] to stimulate proliferation and migration of dermal fibroblasts. In full-thickness excisional wound of diabetic NOD/SCID mice, human AF-MSCs significantly accelerated wound closure through increasing the angiogenic factors, IGF-1, EGF, and interleukin-8 (IL-8), as well as enhancing reepithelialization by expressing keratinocyte-specific proteins and cytokeratin in the wound area [94]. Additionally, in a model of mouse with excisional wound, human AF-MSCs significantly enhanced wound healing via the TGF-beta/SMAD2 pathway [95], while human AF-MSCs accelerated wound closure through TGF- $\beta /$ SMAD2 and PI3K/Akt pathways under the condition of hypoxia [96].

4.5. GMSCs and Wound Healing. Human GMSCs are homogenous, not tumorigenic [97], and easy to be isolated [98] and display stable phenotype. The most significant advantage of human GMSCs is without any ethical problems in clinical application [99]. Moreover, human GMSCs show a greater capacity of proliferation and migration than AMSCs [100] and BM-MSCs without growth factors [99].

In a murine excisional full-thickness skin wound model, systemic infusion of human GMSCs mitigated local inflammation mediated via suppression of inflammatory cells infiltration, production of IL- 6 and TNF- $\alpha$, and increasing expression of interleukin-10 (IL-10) [101]. This mechanism also existed in the hypoxic environment [102]. In addition, human GMSCs have elicited M2 polarization of macrophages, which may contribute to rapid reepithelialization, improvement of angiogenesis, and tissue remodeling of skin wound [101]. 


\section{Are Autologous or Allogeneic MSCs More Appropriate?}

It has been shown that autologous BM-MSCs are a major source and have obvious efficacy in cell therapy for patients suffering from DFU. Most recently, a study on the feasibility of autologous stem cell therapy in diabetic patients showed that AMSCs isolated from distal limbs of diabetic patients with critical ischemia was not satisfactory as an autologous AMSC source because of its improper phenotype and function [103]. In line with above evidence, the initial viability of the mouse MSCs extracted from the bone marrow of diabetic mice was poor in a normal glucose environment in vitro, but the expansion of that was subsequently improved [61].

Although allogeneic MSCs have had potent immunosuppressive properties, evidence also suggests that they elicit potential as a new therapeutic strategy for the treatment of DFU in animal models. Moreover, allogeneic UCB-MSCs have been successfully used to treat patients with DFU. With increasing number of clinical trials of allogeneic MSCs for acute and chronic diseases [104-107], a comprehensive understanding of the difference in immunological profile is essential.

Hence, the potential for autologous or allogeneic MSCs to be used to improve diabetic wound healing appears particularly promising. However, so far preclinical and clinical data are quite limited and further studies need to be explored for the feasibility of autologous and allogeneic MSCs therapy of DFU.

\section{The Further Treatment for DFU}

Recent studies showed that a transgenic L. sericata larvae could secrete platelet derived growth factor-BB (PDGF-BB), a dimeric peptide growth factor that could bind to the platelet derived growth factor (PDGF) receptor and stimulate cell proliferation and survival, and, hence, promote wound healing. It may be a cost-effective manner for nonhealing wounds, especially for patients with DFU [108] and be employed in regenerative medicine strategies to enhance tissue repair.

\section{Conclusion}

A variety of clinical applications need large number of functionally competent MSCs with stable phenotype to achieve successful results.

From the above, the morphology, size, and surface phenotype of MSCs derived from different tissues have no significant difference. Besides BM-MSCs, others possess rich source and greater proliferation capability and can be easily isolated. In addition, UC-MSCs and human AF-MSCs have lower immunogenicity, while AMSCs and human GMSCs pose fewer ethical problems. Although BM-MSCs have some limitations, they are firstly discovered and deeply studied in many clinical trials with satisfactory clinical efficacy. This paper supports the potential of BM-MSCs for treatment of DFU, and it may be the optimal cell type for safe and feasible transplantation of DFU.

\section{Conflicts of Interest}

The authors declare that there are no conflicts of interest regarding the publication of this paper.

\section{Acknowledgments}

This paper was funded by Scientific Foundation of the Science Technology Department of Jilin Province (3D515N603428) and Scientific Foundation of the Ministry of Education, Science and Technology Development Center (3M213BR43428).

\section{References}

[1] W. Yang, J. Lu, J. Weng et al., "Prevalence of diabetes among men and women in China," The New England Journal of Medicine, vol. 362, no. 12, pp. 1090-1101, 2010.

[2] A. Ramachandran, C. Snehalatha, A. S. Shetty, and A. Nanditha, "Trends in prevalence of diabetes in Asian countries," World Journal of Diabetes, vol. 3, pp. 110-117, 2012.

[3] Global report on diabetes, World Health Organization, Geneva, Switzerland, 2016.

[4] D. R. Whiting, L. Guariguata, C. Weil, and J. Shaw, "IDF diabetes atlas: global estimates of the prevalence of diabetes for 2011 and 2030," Diabetes Research and Clinical Practice, vol. 94, no. 3, pp. 311-321, 2011.

[5] M. Brod, A. Nikolajsen, J. Weatherall, and K. M. Pfeiffer, "The economic burden of post-prandial hyperglycemia (PPH) among people with type 1 and type 2 diabetes in three countries," Diabetes Therapy, vol. 7, no. 1, pp. 75-90, 2016.

[6] American Diabetes Association, "Economic costs of diabetes in the U.S. in 2012," Diabetes Care, vol. 36, no. 4, pp. 1033-1046, 2013.

[7] M. M. Iversen, K. Midthjell, G. S. Tell et al., "The association between history of diabetic foot ulcer, perceived health and psychological distress: The Nord-Trøndelag Health Study," BMC Endocrine Disorders, vol. 9, article 18, 2009.

[8] H. Shahbazian, L. Yazdanpanah, and S. M. Latifi, "Risk assessment of patients with diabetes for foot ulcers according to risk classification consensus of International Working Group on Diabetic Foot (IWGDF)," Pakistan Journal of Medical Sciences, vol. 29, no. 3, pp. 730-734, 2013.

[9] Y. Jiang, X. Ran, L. Jia et al., "Epidemiology of type 2 diabetic foot problems and predictive factors for amputation in china," International Journal of Lower Extremity Wounds, vol. 14, no. 1, pp. 19-27, 2015.

[10] C. W. Hicks, S. Selvarajah, N. Mathioudakis et al., "Burden of infected diabetic foot ulcers on hospital admissions and costs," Annals of Vascular Surgery, 2015.

[11] K. K. Evans, C. E. Attinger, A. Al-Attar et al., "The importance of limb preservation in the diabetic population," Journal of Diabetes and its Complications, vol. 25, no. 4, pp. 227-231, 2011.

[12] H. Lin, H. Ouyang, J. Zhu et al., "Lens regeneration using endogenous stem cells with gain of visual function," Nature, vol. 531, no. 7594, pp. 323-328, 2016.

[13] A. A. Kramerov and A. V. Ljubimov, "Stem cell therapies in the treatment of diabetic retinopathy and keratopathy," Experimental Biology and Medicine, vol. 241, no. 6, pp. 559-568, 2016. 
[14] X. Bai, Y. Yan, Y.-H. Song et al., "Both cultured and freshly isolated adipose tissue-derived stem cells enhance cardiac function after acute myocardial infarction," European Heart Journal, vol. 31, no. 4, pp. 489-501, 2010.

[15] K. Sejpal, M. H. Ali, S. Maddileti et al., "Cultivated limbal epithelial transplantation in children with ocular surface burns," JAMA Ophthalmology, vol. 131, no. 6, pp. 731-736, 2013.

[16] S. Basu, H. Ali, and V. S. Sangwan, "Clinical outcomes of repeat autologous cultivated limbal epithelial transplantation for ocular surface burns," American Journal of Ophthalmology, vol. 153, no. 4, pp. 643-650, 2012.

[17] S. Cai, Y. Pan, B. Han, T.-Z. Sun, Z.-Y. Sheng, and X.-B. Fu, "Transplantation of human bone marrow-derived mesenchymal stem cells transfected with ectodysplasin for regeneration of sweat glands," Chinese Medical Journal, vol. 124, no. 15, pp. 2260-2268, 2011.

[18] L. Liu, Y. Yu, Y. Hou et al., "Human umbilical cord mesenchymal stem cells transplantation promotes cutaneous wound healing of severe burned rats," PLoS ONE, vol. 9, no. 2, Article ID e88348, 2014.

[19] O. Lindvall, "Clinical translation of stem cell transplantation in Parkinson's disease," Journal of Internal Medicine, vol. 279, no. 1, pp. 30-40, 2016.

[20] C. Nicoleau, P. Viegas, M. Peschanski, and A. L. Perrier, "Human pluripotent stem cell therapy for Huntington's disease: technical, immunological, and safety challenges," Neurotherapeutics, vol. 8, no. 4, pp. 562-576, 2011.

[21] Q.-S. Zhao, N. Xia, N. Zhao et al., "Localization of human mesenchymal stem cells from umbilical cord blood and their role in repair of diabetic foot ulcers in rats," International Journal of Biological Sciences, vol. 10, no. 1, pp. 80-89, 2013.

[22] J. Wan, L. Xia, W. Liang, Y. Liu, and Q. Cai, “Transplantation of bone marrow-derived mesenchymal stem cells promotes delayed wound healing in diabetic rats," Journal of Diabetes Research, vol. 2013, Article ID 647107, 11 pages, 2013.

[23] L. Rodriguez-Menocal, S. Shareef, M. Salgado, A. Shabbir, and E. Van Badiavas, "Role of whole bone marrow, whole bone marrow cultured cells, and mesenchymal stem cells in chronic wound healing," Stem Cell Research and Therapy, vol. 6, article 24, 2015.

[24] A. I. Arno, S. Amini-Nik, P. H. Blit et al., "Human Wharton's jelly mesenchymal stem cells promote skin wound healing through paracrine signaling," Stem Cell Research and Therapy, vol. 5, no. 1, article no. 28, 2014.

[25] S.-H. Kang, Y.-G. Chung, I.-H. Oh, Y.-S. Kim, K.-O. Min, and J.-Y. Chung, "Bone regeneration potential of allogeneic or autogeneic mesenchymal stem cells loaded onto cancellous bone granules in a rabbit radial defect model," Cell and Tissue Research, vol. 355, no. 1, pp. 81-88, 2014.

[26] L. F. Mellor, M. Mohiti-Asli, J. Williams et al., "Extracellular calcium modulates chondrogenic and osteogenic differentiation of human adipose-derived stem cells: a novel approach for osteochondral tissue engineering using a single stem cell source," Tissue Engineering, vol. 21, no. 17-18, pp. 2323-2333, 2015.

[27] H. E. McCarthy, J. J. Bara, K. Brakspear, S. K. Singhrao, and C. W. Archer, "The comparison of equine articular cartilage progenitor cells and bone marrow-derived stromal cells as potential cell sources for cartilage repair in the horse," Veterinary Journal, vol. 192, no. 3, pp. 345-351, 2012.

[28] A. Technau, K. Froelich, R. Hagen, and N. Kleinsasser, "Adipose tissue-derived stem cells show both immunogenic and immunosuppressive properties after chondrogenic differentiation," Cytotherapy, vol. 13, no. 3, pp. 310-317, 2011.

[29] C. Xia and J. Cao, "Imaging the survival and utility of predifferentiated allogeneic MSC in ischemic heart," Biochemical and Biophysical Research Communications, vol. 438, no. 2, pp. 382-387, 2013.

[30] S. E. Cho, Y. M. Kim, J. S. Jeong, and Y. K. Seo, “The effect of ultrasound for increasing neural differentiation in hBMMSCs and inducing neurogenesis in ischemic stroke model," Life Sciences, vol. 165, pp. 35-42, 2016.

[31] Y. C.-Y. Hsuan, C.-H. Lin, C.-P. Chang, and M.-T. Lin, "Mesenchymal stem cell-based treatments for stroke, neural trauma, and heat stroke," Brain and Behavior, vol. 6, Article ID e00526, 2016.

[32] C. Grégoire, C. Lechanteur, A. Briquet et al., "Review article: mesenchymal stromal cell therapy for inflammatory bowel diseases," Alimentary Pharmacology \& Therapeutics, vol. 45, no. 2, pp. 205-221, 2017.

[33] J. Kato, H. Kamiya, T. Himeno et al., "Mesenchymal stem cells ameliorate impaired wound healing through enhancing keratinocyte functions in diabetic foot ulcerations on the plantar skin of rats," Journal of Diabetes and its Complications, vol. 28, no. 5, pp. 588-595, 2014.

[34] D. Lu, B. Chen, Z. Liang et al., "Comparison of bone marrow mesenchymal stem cells with bone marrow-derived mononuclear cells for treatment of diabetic critical limb ischemia and foot ulcer: a double-blind, randomized, controlled trial," Diabetes Research and Clinical Practice, vol. 92, no. 1, pp. 26-36, 2011.

[35] P. Charbord, "Bone marrow mesenchymal stem cells: historical overview and concepts," Human Gene Therapy, vol. 21, no. 9, pp. 1045-1056, 2010.

[36] F. Mosna, L. Sensebé, and M. Krampera, "Human bone marrow and adipose tissue mesenchymal stem cells: a user's guide," Stem Cells and Development, vol. 19, no. 10, pp. 1449-1470, 2010.

[37] K. Bieback and H. Klüter, "Mesenchymal stromal cells from umbilical cord blood," Current Stem Cell Research and Therapy, vol. 2, no. 4, pp. 310-323, 2007.

[38] M. Jäger, C. Zilkens, B. Bittersohl, and R. Krauspe, "Cord blood-an alternative source for bone regeneration," Stem Cell Reviews and Reports, vol. 5, no. 3, pp. 266-277, 2009.

[39] S. Barlow, G. Brooke, K. Chatterjee et al., "Comparison of human placenta- and bone marrow-derived multipotent mesenchymal stem cells," Stem Cells and Development, vol. 17, no. 6, pp. 1095-1107, 2008.

[40] X. Li, W. Ling, A. Pennisi et al., "Human placenta-derived adherent cells prevent bone loss, stimulate bone formation, and suppress growth of multiple myeloma in bone," Stem Cells, vol. 29, no. 2, pp. 263-273, 2011.

[41] A. Wilson, P. E. Butler, and A. M. Seifalian, "Adipose-derived stem cells for clinical applications: a review," Cell Proliferation, vol. 44, no. 1, pp. 86-98, 2011.

[42] H. J. Jin, Y. K. Bae, M. Kim et al., "Comparative analysis of human mesenchymal stem cells from bone marrow, adipose tissue, and umbilical cord blood as sources of cell therapy," International Journal of Molecular Sciences, vol. 14, no. 9, pp. 17986-18001, 2013.

[43] M. Parvizi and M. C. Harmsen, "Therapeutic prospect of adipose-derived stromal cells for the treatment of abdominal aortic aneurysm," Stem Cells and Development, vol. 24, no. 13, pp. 1493-1505, 2015. 
[44] L. Tang, N. Li, H. Xie, and Y. Jin, "Characterization of mesenchymal stem cells from human normal and hyperplastic gingiva," Journal of Cellular Physiology, vol. 226, no. 3, pp. 832-842, 2011.

[45] F. Wang, M. Yu, X. Yan et al., "Gingiva-derived mesenchymal stem cell-mediated therapeutic approach for bone tissue regeneration," Stem Cells and Development, vol. 20, no. 12, pp. 20932102, 2011.

[46] K. Marynka-Kalmani, S. Treves, M. Yafee et al., "The lamina propria of adult human oral mucosa harbors a novel stem cell population," Stem Cells, vol. 28, no. 5, pp. 984-995, 2010.

[47] Q. You, L. Cai, J. Zheng, X. Tong, D. Zhang, and Y. Zhang, "Isolation of human mesenchymal stem cells from thirdtrimester amniotic fluid," International Journal of Gynecology and Obstetrics, vol. 103, no. 2, pp. 149-152, 2008.

[48] S.-G. Kang, N. Shinojima, A. Hossain et al., "Isolation and perivascular localization of mesenchymal stem cells from mouse brain," Neurosurgery, vol. 67, no. 3, pp. 711-720, 2010.

[49] K. Le Blanc, "Immunomodulatory effects of fetal and adult mesenchymal stem cells," Cytotherapy, vol. 5, no. 6, pp. 485-489, 2003.

[50] M. D. Nicola, C. Carlo-Stella, M. Magni et al., "Human bone marrow stromal cells suppress T-lymphocyte proliferation induced by cellular or nonspecific mitogenic stimuli," Blood, vol. 99, no. 10, pp. 3838-3843, 2002.

[51] M. Krampera, L. Cosmi, R. Angeli et al., "Role for interferon$\gamma$ in the immunomodulatory activity of human bone marrow mesenchymal stem cells," Stem Cells, vol. 24, no. 2, pp. 386-398, 2006.

[52] J. Hua, J. Gong, H. Meng et al., "Comparison of different methods for the isolation of mesenchymal stem cells from umbilical cord matrix: proliferation and multilineage differentiation as compared to mesenchymal stem cells from umbilical cord blood and bone marrow," Cell Biology International, vol. 38, no. 2, pp. 198-210, 2014.

[53] M. Sasaki, R. Abe, Y. Fujita, S. Ando, D. Inokuma, and H. Shimizu, "Mesenchymal stem cells are recruited into wounded skin and contribute to wound repair by transdifferentiation into multiple skin cell type," Journal of Immunology, vol. 180, no. 4, pp. 2581-2587, 2008.

[54] A. H. Amin, Z. Y. Abd Elmageed, D. Nair et al., "Modified multipotent stromal cells with epidermal growth factor restore vasculogenesis and blood flow in ischemic hind-limb of type II diabetic mice," Laboratory Investigation, vol. 90, no. 7, pp. 985996, 2010.

[55] A. O’Loughlin, M. Kulkarni, M. Creane et al., "Topical administration of allogeneic mesenchymal stromal cells seeded in a collagen scaffold augments wound healing and increases angiogenesis in the diabetic rabbit ulcer," Diabetes, vol. 62, no. 7, pp. 2588-2594, 2013.

[56] L. Shen, W. Zeng, Y.-X. Wu et al., "Neurotrophin-3 accelerates wound healing in diabetic mice by promoting a paracrine response in mesenchymal stem cells," Cell Transplantation, vol. 22, no. 6, pp. 1011-1021, 2013.

[57] C. Hou, L. Shen, Q. Huang et al., "The effect of heme oxygenase1 complexed with collagen on MSC performance in the treatment of diabetic ischemic ulcer," Biomaterials, vol. 34, no. 1, pp. 112-120, 2013.

[58] M. Li, Y. Zhao, H. Hao et al., "Mesenchymal stem cellconditioned medium improves the proliferation and migration of keratinocytes in a diabetes-like microenvironment," International Journal of Lower Extremity Wounds, vol. 14, no. 1, pp. 7386, 2015.
[59] S. Iwamoto, X. Lin, R. Ramirez et al., "Bone marrow cell mobilization by the systemic use of granulocyte colony-stimulating factor (GCSF) improves wound bed preparation," International Journal of Lower Extremity Wounds, vol. 12, no. 4, pp. 256-264, 2013.

[60] K. Tatsumi, H. Otani, D. Sato et al., "Granulocyte-colony stimulating factor increases donor mesenchymal stem cells in bone marrow and their mobilization into peripheral circulation but does not repair dystrophic heart after bone marrow transplantation," Circulation Journal, vol. 72, no. 8, pp. 1351-1358, 2008.

[61] L. Shin and D. A. Peterson, "Impaired therapeutic capacity of autologous stem cells in a model of type 2 diabetes," Stem Cells Translational Medicine, vol. 1, no. 2, pp. 125-135, 2012.

[62] W. N. Nowak, S. Borys, K. Kusińska et al., "Number of circulating pro-angiogenic cells, growth factor and anti-oxidative gene profiles might be altered in type 2 diabetes with and without diabetic foot syndrome," Journal of Diabetes Investigation, vol. 5, no. 1, pp. 99-107, 2014.

[63] M. Dominici, K. Le Blanc, I. Mueller et al., "Minimal criteria for defining multipotent mesenchymal stromal cells. The International Society for Cellular Therapy position statement," Cytotherapy, vol. 8, no. 4, pp. 315-317, 2006.

[64] N. R. Dash, S. N. Dash, P. Routray, S. Mohapatra, and P. C. Mohapatra, "Targeting nonhealing ulcers of lower extremity in human through autologous bone marrow-derived mesenchymal stem cells," Rejuvenation Research, vol. 12, no. 5, pp. 359366, 2009.

[65] B. Amann, C. Luedemann, R. Ratei, and J. A. Schmidt-Lucke, "Autologous bone marrow cell transplantation increases leg perfusion and reduces amputations in patients with advanced critical limb ischemia due to peripheral artery disease," Cell Transplantation, vol. 18, no. 3, pp. 371-380, 2009.

[66] V. Procházka, J. Gumulec, F. Jalůvka et al., "Cell therapy, a new standard in management of chronic critical limb ischemia and foot ulcer," Cell Transplantation, vol. 19, no. 11, pp. 1413-1424, 2010.

[67] J. Vojtassak, L. Danisovic, M. Kubes et al., "Autologous biograft and mesenchymal stem cells in treatment of the diabetic foot," Neuroendocrinology Letters, vol. 27, supplement 2, pp. 134-137, 2006.

[68] M. Pietilä, S. Palomäki, S. Lehtonen et al., "Mitochondrial function and energy metabolism in umbilical cord blood- and bone marrow-derived mesenchymal stem cells," Stem Cells and Development, vol. 21, no. 4, pp. 575-588, 2012.

[69] K. Bieback, S. Kern, H. Klüter, and H. Eichler, "Critical parameters for the isolation of mesenchymal stem cells from umbilical cord blood," STEM CELLS, vol. 22, no. 4, pp. 625-634, 2004.

[70] N. Xia, J.-M. Xu, N. Zhao, Q.-S. Zhao, M. Li, and Z.-F. Cheng, "Human mesenchymal stem cells improve the neurodegeneration of femoral nerve in a diabetic foot ulceration rats," Neuroscience Letters, vol. 597, pp. 84-89, 2015.

[71] H.-J. You, S. Namgoong, S.-K. Han, S.-H. Jeong, E.-S. Dhong, and W.-K. Kim, "Wound-healing potential of human umbilical cord blood-derived mesenchymal stromal cells in vitro-a pilot study," Cytotherapy, vol. 17, no. 11, pp. 1506-1513, 2015.

[72] X.-Y. Li, Z.-H. Zheng, X.-Y. Li et al., "Treatment of foot disease in patients with type 2 diabetes mellitus using human umbilical cord blood mesenchymal stem cells: response and correction of immunological anomalies," Current Pharmaceutical Design, vol. 19, no. 27, pp. 4893-4899, 2013. 
[73] S.-S. Yang, N.-R. Kim, K.-B. Park et al., "A phase I study of human cord blood-derived mesenchymal stem cell therapy in patients with peripheral arterial occlusive disease," International Journal of Stem Cells, vol. 6, no. 1, pp. 37-44, 2013.

[74] S. Kern, H. Eichler, J. Stoeve, H. Klüter, and K. Bieback, "Comparative analysis of mesenchymal stem cells from bone marrow, umbilical cord blood, or adipose tissue," Stem Cells, vol. 24, no. 5, pp. 1294-1301, 2006.

[75] Y.-R. Kuo, C.-T. Wang, J.-T. Cheng, G.-S. Kao, Y.-C. Chiang, and C.-J. Wang, "Adipose-derived stem cells accelerate diabetic wound healing through the induction of autocrine and paracrine effects," Cell Transplantation, vol. 25, no. 1, pp. 71-81, 2016.

[76] Y. Kato, T. Iwata, S. Morikawa, M. Yamato, T. Okano, and Y. Uchigata, "Allogeneic transplantation of an adipose-derived stem cell sheet combined with artificial skin accelerates wound healing in a rat wound model of type 2 diabetes and obesity," Diabetes, vol. 64, no. 8, pp. 2723-2734, 2015.

[77] P. J. Amos, S. K. Kapur, P. C. Stapor et al., "Human adiposederived stromal cells accelerate diabetic wound healing: impact of cell formulation and delivery," Tissue Engineering-Part A, vol. 16, no. 5, pp. 1595-1606, 2010.

[78] S. E. Navone, L. Pascucci, M. Dossena et al., "Decellularized silk fibroin scaffold primed with adipose mesenchymal stromal cells improves wound healing in diabetic mice," Stem Cell Research and Therapy, vol. 5, no. 1, article no. 7, 2014.

[79] M. Kadivar, S. Khatami, Y. Mortazavi, M. A. Shokrgozar, M. Taghikhani, and M. Soleimani, "In vitro cardiomyogenic potential of human umbilical vein-derived mesenchymal stem cells," Biochemical and Biophysical Research Communications, vol. 340, no. 2, pp. 639-647, 2006.

[80] X. L. Wang, P. Hu, X. R. Guo et al., "Reprogramming human umbilical cord mesenchymal stromal cells to islet-like cells with the use of in vitro-synthesized pancreatic-duodenal homebox 1 messenger RNA," Cytotherapy, vol. 16, no. 11, pp. 1519-1527, 2014.

[81] L. Ma, B.-L. Cui, X.-Y. Feng et al., "Biological characteristics of human umbilical cord-derived mesenchymal stem cells and their differentiation into neurocyte-like cells," Chinese Journal of Pediatrics, vol. 44, no. 7, pp. 513-517, 2006.

[82] R. Mahmood, M. S. Choudhery, A. Mehmood, S. N. Khan, and S. Riazuddin, "In vitro differentiation potential of human placenta derived cells into skin cells," Stem Cells International, vol. 2015, Article ID 841062, 11 pages, 2015.

[83] D. Baksh, R. Yao, and R. S. Tuan, "Comparison of proliferative and multilineage differentiation potential of human mesenchymal stem cells derived from umbilical cord and bone marrow," Stem Cells, vol. 25, no. 6, pp. 1384-1392, 2007.

[84] S. Karahuseyinoglu, O. Cinar, E. Kilic et al., "Biology of stem cells in human umbilical cord stroma: in situ and in vitro surveys," Stem Cells, vol. 25, no. 2, pp. 319-331, 2007.

[85] Y. Wang, Q.-Q. Dan, Q.-P. Wang et al., "Human umbilic mesenchymal stromal cells repairs diabetic foot in rats associated with VEGF expressional change," Sichuan Da Xue Xue Bao Yi Xue Ban, vol. 45, no. 1, pp. 29-33, 2014.

[86] C. Shrestha, L. Zhao, K. Chen, H. He, and Z. Mo, "Enhanced healing of diabetic wounds by subcutaneous administration of human umbilical cord derived stem cells and their conditioned media," International Journal of Endocrinology, vol. 2013, Article ID 592454, 10 pages, 2013.

[87] N. Zhou, Q. P. Wang, X. F. Jin et al., "Effect of human umbilici mesenchymal stromal cells implantation on the BDNF expression in diabetic foot rats," Sichuan Da Xue Xue Bao Yi Xue Ban, vol. 44, pp. 931-934, 2013.

[88] N. E. Timmins, M. Kiel, M. Günther et al., "Closed system isolation and scalable expansion of human placental mesenchymal stem cells," Biotechnology and Bioengineering, vol. 109, no. 7, pp. 1817-1826, 2012.

[89] S. H. Abd-Allah, A. S. El-Shal, S. M. Shalaby, E. Abd-Elbary, N. F. Mazen, and R. R. Abdel Kader, "The role of placenta-derived mesenchymal stem cells in healing of induced full-thickness skin wound in a mouse model," IUBMB Life, vol. 67, no. 9, pp. 701-709, 2015.

[90] P. Kong, X. Xie, F. Li, Y. Liu, and Y. Lu, "Placenta mesenchymal stem cell accelerates wound healing by enhancing angiogenesis in diabetic Goto-Kakizaki (GK) rats," Biochemical and Biophysical Research Communications, vol. 438, no. 2, pp. 410-419, 2013.

[91] P. De Coppi, G. Bartsch Jr., M. M. Siddiqui et al., "Isolation of amniotic stem cell lines with potential for therapy," Nature Biotechnology, vol. 25, no. 1, pp. 100-106, 2007.

[92] E. C. Moorefield, E. E. McKee, L. Solchaga et al., "Cloned, CD117 selected human amniotic fluid stem cells are capable of modulating the immune response," PLoS ONE, vol. 6, no. 10, Article ID e26535, 2011.

[93] A. Skardal, D. Mack, E. Kapetanovic et al., "Bioprinted amniotic fluid-derived stem cells accelerate healing of large skin wounds," Stem Cells Translational Medicine, vol. 1, no. 11, pp. 792-802, 2012.

[94] S.-W. Kim, H.-Z. Zhang, L. Guo, J.-M. Kim, and M. H. Kim, "Amniotic mesenchymal stem cells enhance wound healing in diabetic NOD/SCID mice through high angiogenic and engraftment capabilities," PLoS ONE, vol. 7, no. 7, Article ID e41105, 2012.

[95] B. S. Yoon, J.-H. Moon, E. K. Jun et al., "Secretory profiles and wound healing effects of human amniotic fluid-derived mesenchymal stem cells," Stem Cells and Development, vol. 19, no. 6, pp. 887-902, 2010.

[96] E. K. Jun, Q. Zhang, B. S. Yoon et al., "Hypoxic conditioned medium from human amniotic fluid-derived mesenchymal stem cells accelerates skin wound healing through TGF$\beta /$ SMAD2 and PI3K/AKT pathways," International Journal of Molecular Sciences, vol. 15, no. 1, pp. 605-628, 2014.

[97] S. Santamaría, N. Sanchez, M. Sanz, and J. A. Garcia-Sanz, "Comparison of periodontal ligament and gingiva-derived mesenchymal stem cells for regenerative therapies," Clinical Oral Investigations, pp. 1-8, 2016.

[98] S.-M. Wu, H.-C. Chiu, Y.-T. Chin et al., "Effects of enamel matrix derivative on the proliferation and osteogenic differentiation of human gingival mesenchymal stem cells," Stem Cell Research and Therapy, vol. 5, article 52, 2014.

[99] G. B. Tomar, R. K. Srivastava, N. Gupta et al., "Human gingiva-derived mesenchymal stem cells are superior to bone marrow-derived mesenchymal stem cells for cell therapy in regenerative medicine," Biochemical and Biophysical Research Communications, vol. 393, no. 3, pp. 377-383, 2010.

[100] M. A. Boink, L. J. Van Den Broek, S. Roffel et al., "Different wound healing properties of dermis, adipose, and gingiva mesenchymal stromal cells," Wound Repair and Regeneration, vol. 24, no. 1, pp. 100-109, 2016.

[101] Q.-Z. Zhang, W.-R. Su, S.-H. Shi et al., "Human gingiva-derived mesenchymal stem cells elicit polarization of M2 macrophages and enhance cutaneous wound healing," STEM CELLS, vol. 28, no. 10, pp. 1856-1868, 2010. 
[102] C. M. Jiang, J. Liu, J. Y. Zhao et al., "Effects of hypoxia on the immunomodulatory properties of human gingiva-derived mesenchymal stem cells," Journal of Dental Research, vol. 94, no. 1, pp. 69-77, 2015.

[103] Z. Kočí, K. Turnovcová, M. Dubský et al., "Characterization of human adipose tissue-derived stromal cells isolated from diabetic patient's distal limbs with critical ischemia," Cell Biochemistry and Function, vol. 32, no. 7, pp. 597-604, 2014.

[104] J. Panés, D. García-Olmo, G. Van Assche et al., "Expanded allogeneic adipose-derived mesenchymal stem cells (Cx601) for complex perianal fistulas in Crohn's disease: a phase 3 randomised, double-blind controlled trial," The Lancet, vol. 388, pp. 1281-1290, 2016.

[105] M. E. J. Reinders, G. J. Dreyer, J. R. Bank et al., "Safety of allogeneic bone marrow derived mesenchymal stromal cell therapy in renal transplant recipients: the neptune study," Journal of Translational Medicine, vol. 13, article 344, 2015.

[106] A. Can, A. T. Ulus, O. Cinar et al., "Human umbilical cord mesenchymal stromal cell transplantation in myocardial ischemia (HUC-HEART trial). A study protocol of a phase $1 / 2$, controlled and randomized trial in combination with coronary artery bypass grafting," Stem Cell Reviews and Reports, vol. 11, no. 5, pp. 752-760, 2015.

[107] A. Vega, M. A. Martín-Ferrero, F. Del Canto et al., "Treatment of knee osteoarthritis with allogeneic bone marrow mesenchymal stem cells: a randomized controlled trial," Transplantation, vol. 99, no. 8, pp. 1681-1690, 2015.

[108] R. J. Linger, E. J. Belikoff, Y. Yan et al., “Towards next generation maggot debridement therapy: transgenic Lucilia sericata larvae that produce and secrete a human growth factor," BMC Biotechnology, vol. 16, no. 1, article 30, 2016. 


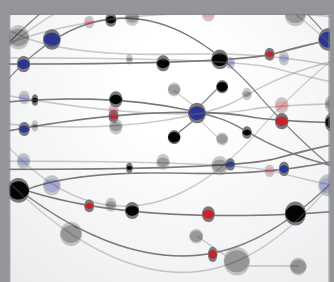

The Scientific World Journal
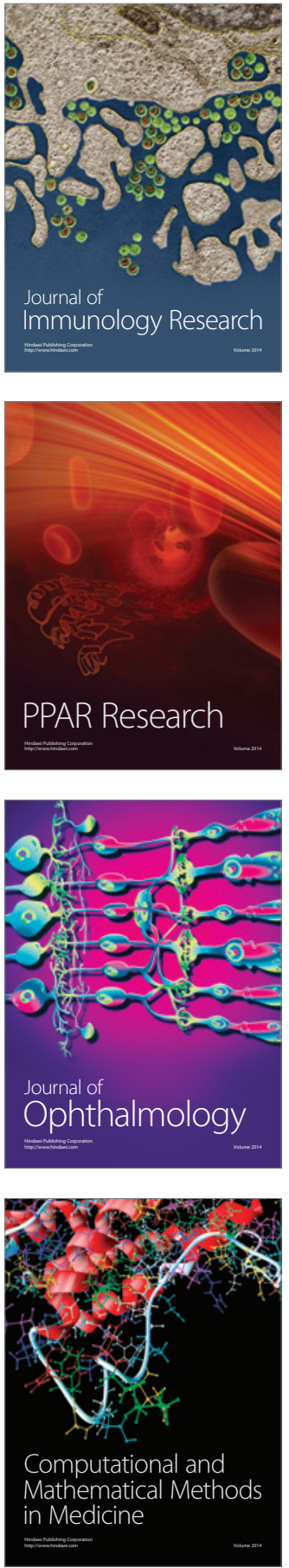

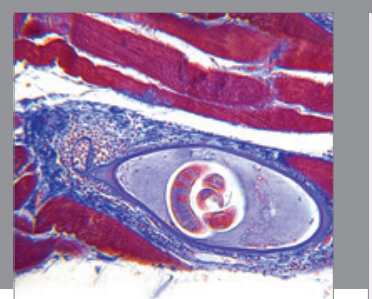

Gastroenterology Research and Practice
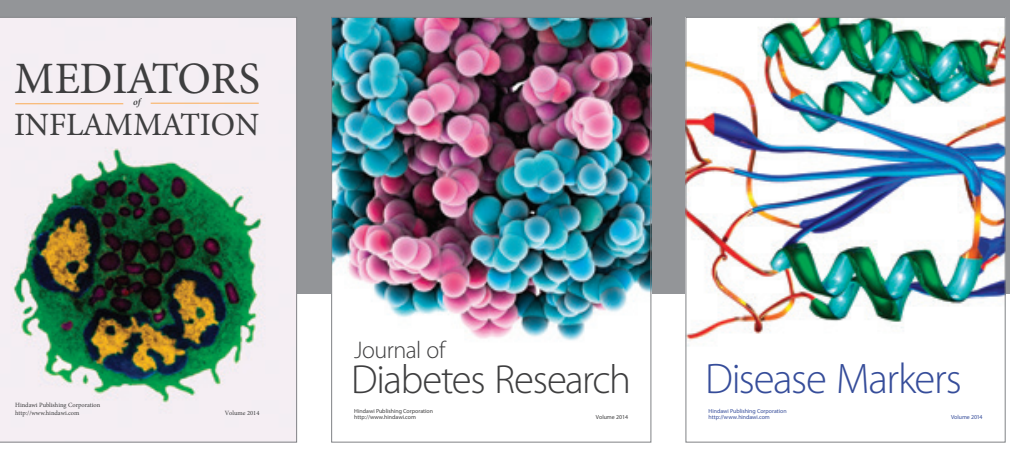

Disease Markers

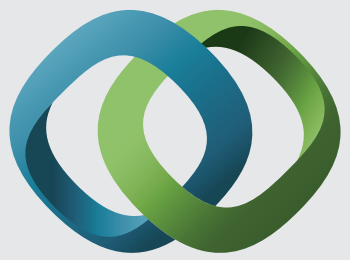

\section{Hindawi}

Submit your manuscripts at

https://www.hindawi.com
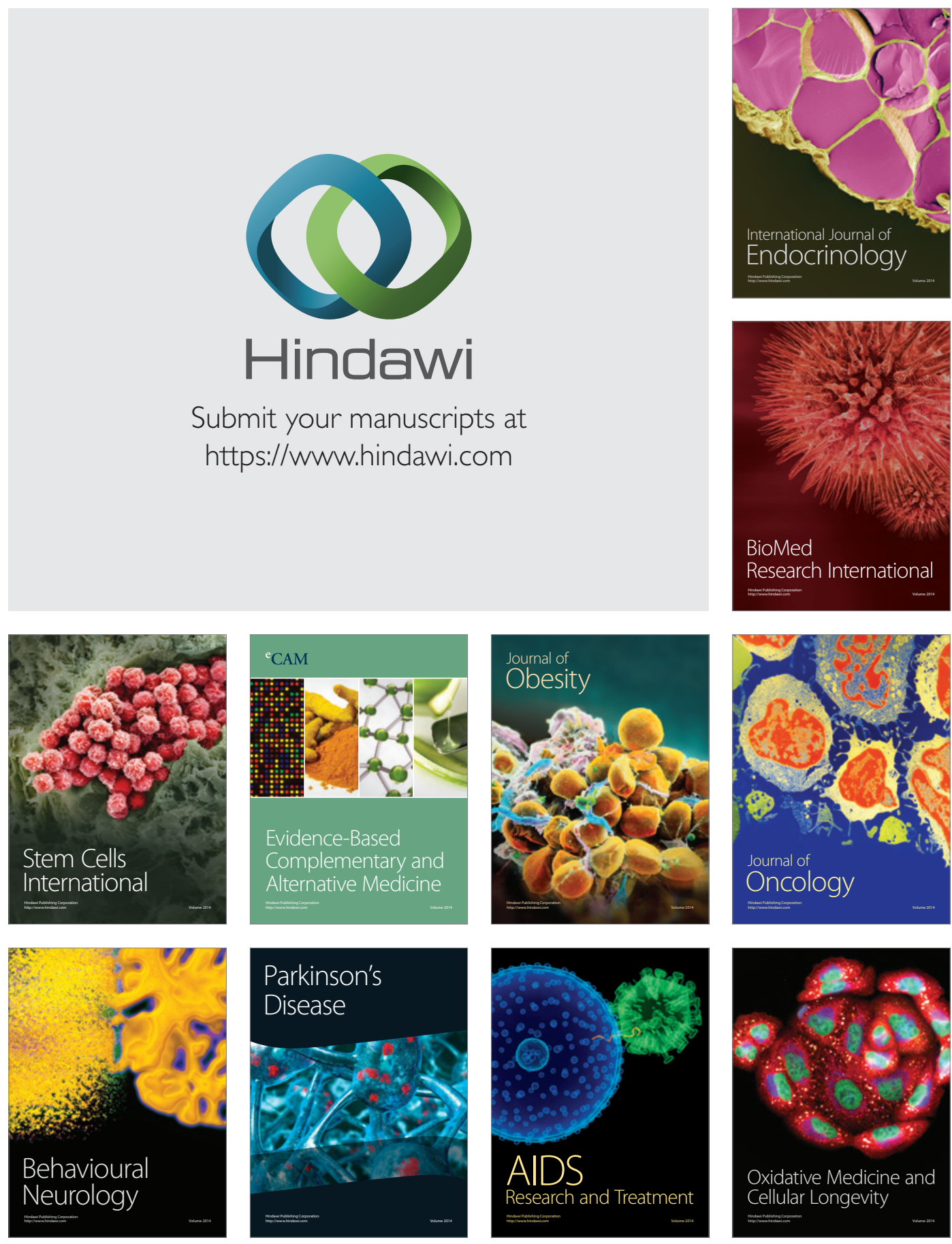